\title{
Biting midges (Diptera: Ceratopogonidae) from Martín García Island, Argentina
}

\author{
María M. Ronderos, Pablo I. Marino, Florentina Díaz \& Ana L. Estévez \\ División Entomología, Museo de La Plata, Paseo del Bosque s/n, 1900 La Plata, Argentina; \\ ronderos@fcnym.unlp.edu.ar, pmarino@fcnym.unlp.edu.ar, mfdiaz@fcnym.unlp.edu.ar, \\ anitaestevez@argentina.com
}

Received 21-VII-2010. C Corrected 04-I-2011. Accepted 01-II-2011.

\begin{abstract}
Nearly 230 species of biting midges have been recorded or described from Argentina; 38 of them are known from the Buenos Aires province and only one is cited from Martín García Island. This paper presents the results raised from six collecting trips which took place on the island during spring 2005, summer 2006 and autumn 2009. Diverse sampling sites including permanent and temporary aquatic environments were chosen, most of the ten sampling sites were ponds of diverse origin, some of these environments were covered with floating vegetation as Lemna gibba, Lemna minuscule, Salvinia biloba, Salvinia minima, Azolla filiculoides, Limnobium laevigatum, Pistia stratiotes, Spirodela intermedia, Wolffiella oblonga and Wolffia columbiana. Other sites were placed in urban and suburban areas. Adults were collected with sweep nets at sunrise and sunset and with light traps at intervals of four to five hours at night, depending on electricity availability on the island. Larvae and pupae were collected with different implements depending on characteristics of each surveyed aquatic habitat. In free standing water, they were captured with small sieves or hand pipettes and micropipettes, flotation techniques were utilized for sampling vegetated areas, free and rooted floating hydrophytes were extracted for removing insects among them. Thirteen species of Ceratopogonidae were collected, three of Atrichopogon Kieffer, three of Forcipomyia Meigen, two of Dasyhelea Kieffer, four of Culicoides Latreille, and one of Bezzia Kieffer, all representing new records from the island. Rev. Biol. Trop. 59 (3): 1183-1194. Epub 2011 September 01.
\end{abstract}

Key words: biting midges, Ceratopogonidae, new records, Martín García Island, Argentina.

Martín García Island is located in the Northeastern bend of the Río de la Plata estuary, near the mouth of Río Uruguay and the lower delta of Río Paraná (34²10'56" S - 58 $15^{\circ} 86^{\prime}$ " W), in North of Buenos Aires province, Argentina. It is the highest island in the Paraná River delta region, at $27 \mathrm{~m}$ above sea level, with an annual average rainfall of $1000 \mathrm{~mm}$. The island is composed of granite and other igneous intrusive rocks of the Brasilian massif; differs from other islands of Delta del Paraná which were formed by sediments deposited by the Paraná and Uruguay rivers. The origin and resulting geomorphologic features of Martín García have given rise to an unusually varied flora and fauna.
In order to preserve its flora and fauna, the island was declared Provincial Natural Reserve in 1998. Lahitte \& Hurrell (1994, 1996) published studies of trees and bushes as well as aquatic plants. Contributions dealing with vertebrates were provided by Juárez (1995), Lahitte et al. (1995) and Lahitte \& Hurrell (1998). There have been only a few noteworthy contributions on invertebrates, such as those on annelids (Armendáriz \& César 2001, César \& Liberto 2008, César et al. 2001, César et al. 2009) and ostracods (César \& Liberto 2008, César et al. 2001). Except for the excellent works on aquatic and semi aquatic Heteroptera and Coleoptera occurring on the island (Viana 1937, Fernández \& López Ruf 1999) most 
other aquatic and semi-aquatic insect groups have not been adequately studied.

Nearly 230 species of biting midges (Diptera: Ceratopogonidae) have been recorded or described from Argentina (Spinelli 1998, Spinelli \& Wirth 1993, Ronderos \& Spinelli 2002, Borkent \& Spinelli 2007). The ceratopogonid fauna of Buenos Aires province is well known with 38 species recorded, 25 of which belong to Atrichopogon Kieffer, Forcipomyia Meigen, Dasyhelea Kieffer, Culicoides Latreille and Bezzia Kieffer; only one species of Forcipomyia has previously been cited from Martín García Island. Most of these ceratopogonids have been collected during studies of nocturnal activity and the influence of climatic and physical factors on their abundance (Spinelli \& Balseiro 1982, Spinelli et al. 1989). The development of immature stages of four species has also been treated (Ronderos \& Spinelli 2000, Ronderos \& Díaz 2002, Ronderos et al. 2003a, Spinelli et al. 2005a). The larvae and pupae of nearly all members of this family are aquatic or semi-aquatic, detritivores or predators. Adults are herbivorous or predators, sucking nectar from flowers or insects' blood and of other invertebrates and vertebrates. These alimentary habits make these insects renowned all over the world by their economic significance, because many of them are disease vectors or pollinators (Spinelli \& Wirth 1993, Ronderos et al. 2003b).

We present results of field work on Martín García Island, a previously poorly known area in Buenos Aires province, and provide a list of ceratopogonids collected from this island (Table 1).

\section{MATERIAL AND METHODS}

Six collecting trips were conducted on the island during spring 2005 and summer 2006, and autumn 2009. Most of the ten sampling sites were ponds produced by excavations for basalt, natural ponds at the base of a sand dune formed in natural depressions in the marginal forest, or, in zones near the inland sandy plains. These sampling sites were located in xerophytic forest, transition forest, regular forest, sandy soils, urban and suburban areas (Fig. 1). The inland aquatic habitats near river margins were produced by river overflows and rainfall; other marshes were formed by rainfall accumulation. Sampling sites 5 and 7 were considered stable habitats; sampling sites 1-4, 6, 8 and 9 were considered temporary environments and during the sampling period underwent fluctuations of water levels, until desiccation.

Floating vegetation covering these habitats include Lemna gibba L., Lemna minuscule Herter, Salvinia biloba Raddi, Salvinia minima Baker, Azolla filiculoides Lam., Limnobium laevigatum (Hum. \& Boupl. ex Willd.) Heine, Pistia stratiotes L., Spirodela intermedia W. Koch, Wolffiella oblonga (Phil.) Hegelm and Wolffia columbiana $\mathrm{H}$. Karst. Their actual species composition was changeable according to amount and quality of nutrients depending on time of the year (Lahitte \& Hurrell 1996).

Adults were collected with sweep nets at sunrise and sunset and with light traps at intervals of 4 to 5 hours at night, depending on electricity availability on the island. Larvae and pupae were collected with different implements depending on characteristics of each surveyed aquatic habitat. In free standing water, they were captured with small sieves or hand pipettes and micropipettes, flotation techniques

TABLE 1

List of species collected on Martín García Island

\begin{tabular}{lccc}
\multicolumn{1}{c}{ Species } & Adult & Larva & Pupa \\
Atrichopogon albinensis & $\mathrm{x}$ & & \\
Atrichopogon homofacies & $\mathrm{x}$ & & \\
Atrichopogon sp. & & $\mathrm{x}$ & \\
Forcipomyia pictoni (Forcipomyia) & $\mathrm{x}$ & & \\
Forcipomyia poulaineae (Forcipomyia) & $\mathrm{x}$ & & \\
Forcipomyia rioplatensis (Forcipomyia) & $\mathrm{x}$ & & \\
Dasyhelea necrophila & $\mathrm{x}$ & $\mathrm{x}$ & $\mathrm{x}$ \\
Dasyhelea sp. & $\mathrm{x}$ & $\mathrm{x}$ & $\mathrm{x}$ \\
Culicoides caridei & $\mathrm{x}$ & & \\
Culicoides debilipalpis & $\mathrm{x}$ & & \\
Culicoides insignis & $\mathrm{x}$ & & \\
Culicoides venezuelensis & $\mathrm{x}$ & $\mathrm{x}$ & $\mathrm{x}$ \\
Bezzia blantoni & $\mathrm{x}$ & $\mathrm{x}$ & $\mathrm{x}$
\end{tabular}




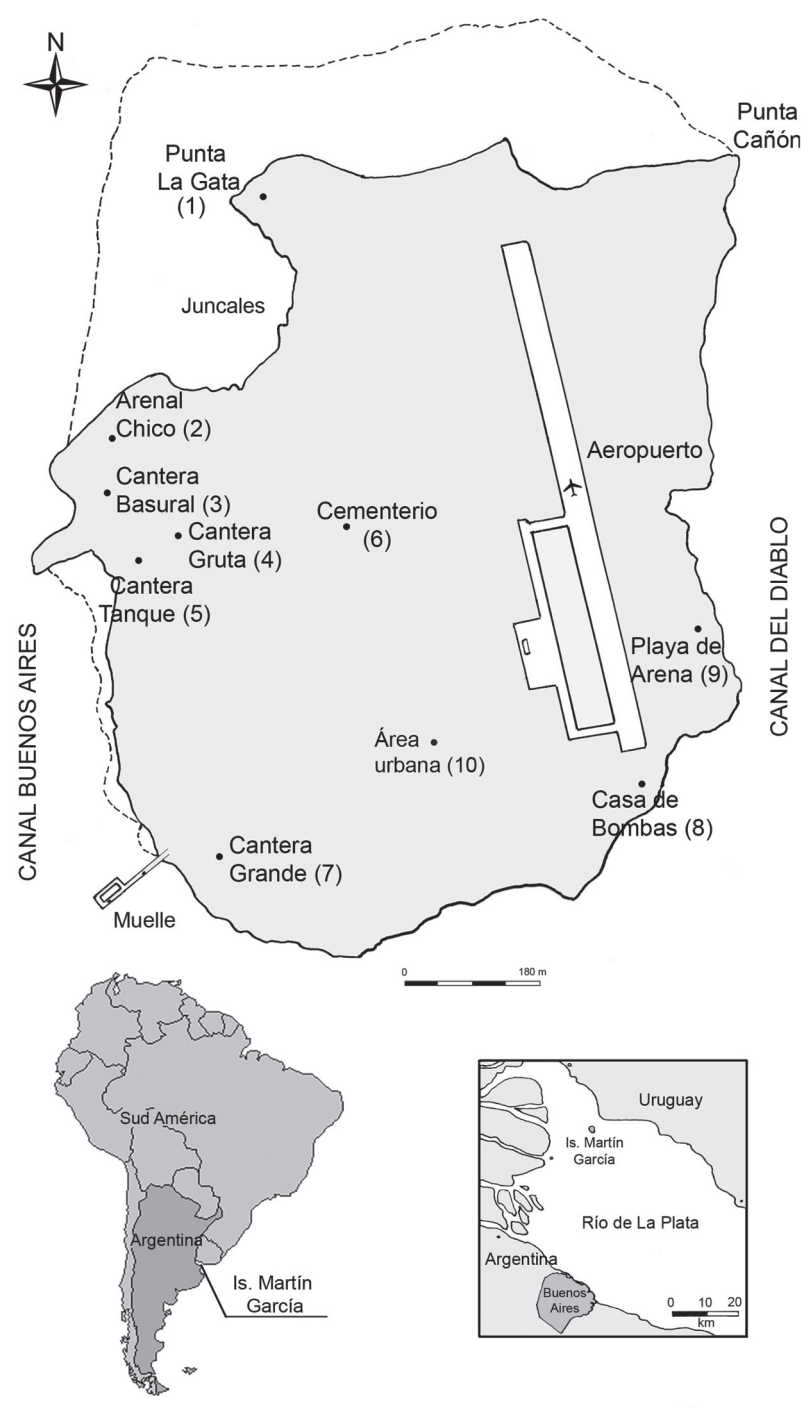

Fig. 1. Map of sampling sites on Isla Martín García. (1) Punta La Gata, (2) Arenal chico, (3) Cantera Basural, (4) Cantera Gruta, (5) Cantera Tanque, (6) Cementerio, (7) Cantera Grande, (8) Casa de Bombas, (9) Playa de arena, (10) Área urbana.

were utilized for sampling vegetated areas, and free and rooted floating hydrophytes were extracted to remove insects among them. Specimens were preserved in $70 \%$ ethyl alcohol, most adults and immature stages were subsequently slide mounted in Canada balsam for microscopic examination and some larvae were reared in the laboratory, in petri dishes containing water and detritus taken from their natural substrates. Pupae were placed in separate vials, with a drop of water until adult emergence.

Specimens were identified with the use of keys (Spinelli et al. 2005, Borkent \& Spinelli 2007) and by comparing them with previously identified material. Voucher specimens are deposited in the collection of the División Entomología, Museo de La Plata, Argentina (MLPA). 
RESULTS

The following 13 species of Ceratopogonidae in Atrichopogon (3 species), Forcipomyia (3 species), Dasyhelea (2 species), Culicoides (4 species) and Bezzia (1 species), were collected during the field work and are new records from Martín García island, Argentina.

\section{Atrichopogon albinensis Ingram \& Macfie}

Atrichopogon albinensis Ingram \& Macfie, 1931: 228 (female; Argentina); Wirth, 1974: 13 (in catalog species south of USA); Spinelli \& Wirth, 1993: 23 (in list of Argentinean species); Borkent \& Wirth, 1997: 22 (in World catalog); Spinelli, 1998: 325 (in list of Argentinean species); Borkent \& Spinelli, 2000: 10 (in catalog species south of USA); Borkent \& Spinelli, 2007: 44 (in Neotropical catalog); Borkent, 2009: 17 (online catalog).

Distribution: Argentina (Buenos Aires).

Material examined: Argentina, Buenos Aires, Isla Martín García: Área urbana, 11-X2006, Ronderos-Díaz col., 1 male, 1 female, at light.

Comments: Atrichopogon albinensis was previously only known from the type locality. Specimens were collected with light trap at sampling site 10 . The immatures are still unknown.

\section{Atrichopogon homofacies Spinelli}

Atrichopogon homofacies Spinelli in Spinelli et al., 1989: 734 (male, female; Argentina); Spinelli \& Wirth, 1993: 23 (in list of Argentinean species); Borkent \& Wirth, 1997: 24 (in World catalog); Spinelli, 1998: 325 (in list of Argentinean species); Borkent \& Spinelli, 2000: 11 (in catalog species south of USA); Borkent \& Spinelli, 2007: 46 (in Neotropical catalog); Borkent, 2009: 21 (online catalog).
Distribution: Argentina (Buenos Aires).

Material examined: Argentina, Buenos Aires, Isla Martín García: Área urbana, 11-X2006, Ronderos-Díaz col., 1 male, 1 female, at light.

Comments: Atrichopogon homofacies was previously only known from the type locality. Specimens were collected with light trap during nights at sampling site 10 . The immatures are still unknown.

\section{Atrichopogon sp.}

Material examined: Argentina, Buenos Aires prov., Isla Martín García, Arenal Chico, 11-XI-2005, Ronderos-Díaz col., 2 larvae; same data except 31-III-2006, 1 larva.

Comments: Immature stages were collected in submerged in muddy water with pipette at sampling site 2 .

\section{Forcipomyia (Forcipomyia) pictoni Macfie}

Forcipomyia pictoni Macfie, 1938: 161 (male; Trinidad).

Forcipomyia (Forcipomyia) pictoni: Wirth, 1974: 6 (in catalog species south of USA); Wirth, 1976: 78 (descr. female, larva, pupa, male redescr.; distrib.); Borkent \& Wirth, 1997: 36 (in World catalog); Marino \& Spinelli, 1999: 446 (in key); Borkent \& Spinelli, 2000: 15 (in catalog species south of USA); Borkent \& Spinelli, 2007: 51 (in Neotropical catalog); Borkent \& Grogan, 2009: 7 (in Nearctic catalog); Borkent, 2009: 38 (online catalog); Spinelli et al. 2010: in press (new records).

Distribution: Widespread, from USA to Brazil (Amazonas, Pará, Bahia and São Paulo) and Argentina (La Rioja, Formosa, Misiones, Corrientes). 
Material examined: Argentina, Buenos Aires, Isla Martín García: Área urbana, 30/31III-2006, Ronderos col., 1 male, at light.

Comments: Specimens were collected with light trap during dusk at sampling site 10 .

\section{Forcipomyia (Forcipomyia) poulaineae Ingram \& Macfie}

Forcipomyia poulaineae Ingram \& Macfie, 1931: 227 (male; Argentina); Macfie, 1939: 142 (in key).

Forcipomyia (Forcipomyia) poulaineae: Wirth, 1974: 6 (in catalog species south of USA); Spinelli, 1983: 124 (female descr.); Spinelli \& Wirth, 1993: 27 (in list of Argentinean species); Borkent \& Wirth, 1997: 36 (in World catalog); Spinelli, 1998: 325 (in list of Argentinean species); Marino \& Spinelli, 1999: 450 (species group); Borkent \& Spinelli, 2000: 15 (in catalog species south of USA); Borkent \& Spinelli, 2007: 51 (in Neotropical catalog); Borkent, 2009: 38 (online catalog).

Distribution: Brazil (Bahía, Santa Catarina), Argentina (Buenos Aires).

Material examined: Argentina, Buenos Aires, Isla Martín García, urban area, 11-X2006, Ronderos-Díaz col., 1 male, at light.

Comments: The specimens were collected with light trap during dusk at sampling site 10 .

\section{Forcipomyia (Forcipomyia) rioplatensis Marino \& Spinelli}

Forcipomyia (Forcipomyia) rioplatensis Marino \& Spinelli, 2002: 314 (male, female; Argentina); Spinelli et al. 2005a: 109 (descr. larva, pupa); Borkent \& Spinelli, 2007: 51 (in Neotropical catalog); Borkent, 2009: 39 (online catalog); Spinelli et al. 2010: in press (new records).

Distribution: Argentina (Buenos Aires, Córdoba), Uruguay.
Material examined: Argentina, Buenos Aires, Isla Martín García, urban area, 30/31III-2006, Ronderos-Díaz col., 1 female, at light; same data except 11-X-2006, 1 male, 3 females.

Comments: The specimens were collected with light trap during dusk at sampling site 10 .

\section{Dasyhelea necrophila Spinelli \& Rodriguez}

Dasyhelea necrophila Spinelli \& Rodriguez, 1999: 59 (male, female, pupa, larva); Ronderos et al. 2003a: 295 (larva, pupa redescr.), Ronderos et al. 2006: 291 (eggs), Borkent \& Spinelli, 2007: 60 (in Neotropical catalog); Borkent, 2009: 67 (online catalog).

Distribution: México, Argentina (Buenos Aires).

Material examined: Argentina, Buenos Aires, Isla Martín García, Cementerio, 12-X2006, Díaz col., 2 males (reared in lab, with larval and pupal exuviae).

Comments: The immature stages were collected from flower pots, with pipette at sampling site 6 .

\section{Dasyhelea sp.}

Material examined: Argentina, Buenos Aires, Isla Martín García, Casa de Bombas, 16-IV-2009, Díaz-Marino col., 3 males, 4 females, (reared in lab, with pupal exuviae), 4 larvae (preserved in alcohol 70\%).

Comments: The immature stages were collected in a small hole filled by overflows of the nearby river with pipette and floating techniques at sampling site 8 . The description of this species will be presented in a future contribution.

\section{Culicoides caridei (Brèthes)}

Cotocripus caridei Brèthes 1912: 451 (female; Argentina). 
Culicoides caridei: Lane, 1945: 366 (redescr., comb.; Brazil); Barbosa, 1947: 13 (Uruguay); Wirth, 1960: 44 (female, redescr.); Cavalieri \& Chiossone, 1966: 147 (Argentina), Wirth, 1974: 29 (in catalog species south of USA); Spinelli, 1982: 80 (redescr.; Argentina); Spinelli \& Wirth, 1986: 59 (in key, distribution); Wirth et al. 1988: 54 (in Neotropical atlas, wing); Ronderos \& Spinelli, 1990: 83 (male); Spinelli \& Wirth, 1993: 34 (in list of Argentinean species); Borkent \& Wirth, 1997: 64 (in World catalog); Spinelli, 1998: 325 (in list of Argentinean species); Borkent \& Spinelli, 2000: 29 (in catalog of south USA species); Ronderos \& Spinelli, 2002: 90 (in key); Spinelli et al. 2005b: 138, 142 (in key, wing photo; Argentina); Spinelli \& Ronderos, 2005: 63 (wing); Borkent \& Spinelli, 2007: 64 (in Neotropical catalog); Borkent, 2009: 78 (online catalog).

Centrorrhynchus setifer Lutz, 1913: 64 (female; Brazil).

Dasyhelea caridei Lane \& Ortiz, 1950: 460 (comb.); Forattini 1957: 189 (generic position).

Distribution: Southeastern Brazil, Uruguay, Argentina (Buenos Aires).

Material examined: Argentina, Buenos Aires prov., Isla Martín García: Área urbana, 9-III-2006, Ronderos col., 5 females, ex man; same data except 10-X-2006, 3 females; same data except Cantera Basural, 11-X-2006, 5 females.

Comments: The specimens were collected biting on humans during dusk at sampling sites 3,10 .

\section{Culicoides debilipalpis Lutz}

Culicoides debilipalpis Lutz, 1913: 60 (female, fig. wing; Brazil); Macfie, 1937: 7 (female; redescr.; Trinidad); Floch \& Abonnenc, 1942: 3 (figs.; Guyane); Barbosa, 1952: 12 (fig.; Argentina, Brazil); Forattini, 1957: 383 (in part.; redescr., figs.; distrib.); Wirth \& Blanton, 1959: 442 (redescr., figs.; Panama);
Franca Rodriguez, 1963: 67 (Uruguay); Messersmith, 1964: 339 (breeding site; Virginia); Smith \& Varnell, 1967: 520 (tree holes; Florida); Wirth \& Blanton, 1971a: 34 (redescr., distrib.); Wirth \& Blanton, 1971b: 75 (khalafi as syn.; notes); Blanton \& Wirth, 1979: 78 (redescr., figs., distrib.); Vitale et al. 1981: 149 (notes; Panama); Spinelli \& Ronderos 1997: 304 (revalidation); Borkent \& Spinelli, 2000: 32 (in catalog species south of USA); Spinelli et al. 2005b: 141, 146 (in key, wing photo; Argentina); Trindade \& Gorayeb, 2005: 67 (records for Para, Brazil); Borkent \& Spinelli, 2007: 66 (in Neotropical catalog); Borkent \& Grogan, 2009:14 (in Nearctic catalog); Borkent, 2009: 80 (online catalog).

Culicoides ichesi Ronderos \& Spinelli, 1995: 77 (female, figs.; Argentina, Paraguay, Uruguay); Ronderos \& Spinelli, 1998: 81 (in key; record for the Yacyreta dam lake area).

Culicoides khalafi Beck, 1957: 104 (male, female, fig.; Florida).

Culicoides lahillei; Spinelli \& Wirth, 1986: 62 (misident., in part., syn. debilipalpis); Wirth et al. 1988: 48 (in Atlas, wing photo.); Spinelli et al. 1989: 735 (in list); Spinelli \& Ronderos, 1991: 91 (record for the Salto Grande dam lake area); Spinelli \& Martinez, 1991: 176 (in key; Uruguay); Waller et al. 1990: 352 (redescr., wing photo; Guyana); Borkent \& Wirth, 1997: 72 (in catalog, syn. debilipalpis).

Distribution: Widespread from USA to Argentina.

Material examined: Argentina, Buenos Aires prov., Isla Martín García, Cantera Basural, 3-III-2006, Ronderos col., 3 females, sweep net (preserved in alcohol 70\%), same data except 30/31-III-2006, 1 female.

Comments: Specimens were collected with sweep net during day time at sampling site 3 .

\section{Culicoides insignis Lutz}

Culicoides insignis Lutz, 1913: 50 (male, female, pupa, fig. wing; Brazil); Costa Lima, 
1937: 415 (fig. palpus); Floch \& Abonnenc, 1942: 1 (fig. wing, palpus; Guyana Francesa); Barbosa, 1947: 20 (fig. male genitalia); Fox, 1948: 25 (notes); Barbosa, 1952: 16 (notes on Lutz collection); Wirth \& Blanton, 1956: 319 (redescr., male lectotype design., distrib.); Forattini et al. 1956: 195 (larva, pupa; Brazil); Forattini, 1957: 223 (redescr., distrib.); Wirth \& Blanton, 1959: 285 (redescr., figs.; Panamá); Williams, 1964: 463 (habitat larva; Trinidad); Linley, 1965: 57 (pupa; Jamaica); Cavalieri \& Chiossone, 1966: 148 (Argentina); Wirth, 1974: 25 (in catalog species south of USA); Wirth \& Blanton, 1974: 57 (redescr.; Antillas); Aitken et al. 1975: 130 (Trinidad); Spinelli \& Wirth, 1986: 52 (in key, wing photo); Wirth et al. 1988: 16 (in Neotropical atlas wing); Spinelli \& Ronderos, 1991: 85 (Uruguay); Spinelli \& Wirth, 1993: 34 (in list of Argentinean species); Borkent \& Wirth, 1997: 71 (in World catalog); Spinelli, 1998: 325 (in list of Argentinean species); Ronderos \& Spinelli, 1998: 79 (in key); Borkent \& Spinelli, 2000: 34 (in catalog species of south USA); Spinelli et al. 2005b: 139, 142 (in key, wing photo; Argentina); Spinelli \& Ronderos, 2005: 63 (wing); Borkent \& Spinelli, 2007: 68 (in Neotropical catalog); Borkent \& Grogan, 2009:14 (in Nearctic catalog); Borkent, 2009: 86 (online catalog).

Culicoides inamollae Fox \& Hoffman, 1944:110 (male, female; Puerto Rico).

Culicoides painteri Fox, 1946: 257 (female; Honduras).

Distribution: USA, México, Central America and Caribean to central Argentina.

Material examined: Argentina, Buenos Aires prov., Isla Martín García, Cantera Tanque, 18/23-X-2006, Ronderos-Díaz col., 1 female, sweep net.

Comments: Specimens were collected with sweep net during day time, at sampling site 5 .

\section{Culicoides venezuelensis}

Ortiz \& Mirsa

Psycophaena pictipennis Philippi, 1865: 628 (female, fig. wing; Chile).

Culicoides venezuelensis Ortiz \& Mirsa, 1950: 137 (male, female, figs.; Venezuela); Wirth, 1955: 234 (= pictipennis Philippi 1865, non Steager, 1839; = ortizi Fox); Forattini, 1957: 463 (redescr.; distrib., figs.); Wirth \& Blanton, 1959: 372 (redescr., figs.; Panama); Wirth, 1974: 36 (in catalog species south of USA); Spinelli \& Wirth, 1986: 64 (in key, distrib., wing photo); Wirth et al. 1988: 42 (in Neotropical atlas, wing); Ronderos \& Spinelli, 1989: 77 (pupa); Spinelli \& Wirth, 1993: 35 (in list of Argentinean species); Borkent \& Wirth, 1997: 84 (in World catalog); Borkent \& Spinelli, 2000: 37 (in catalog species of south USA); Spinelli \& Borkent, 2004: 393 (Costa Rica record); Díaz et al. 2005: 1 (eggs); Spinelli et al. 2005b: 140, 144 (in key, wing photo; Argentina); Borkent \& Spinelli, 2007: 71 (in Neotropical catalog); Borkent, 2009: 104 (online catalog).

Culicoides ortizi Fox, 1952: 366 (female; Venezuela).

Distribution: Costa Rica to Chile and Argentina.

Material examined: Argentina, Buenos Aires prov., Isla Martín García, Arenal Chico, 11-X-2006, Ronderos-Díaz col., 1 male, 1 female, sweep net; same data except 18/23-X2006, 1 female (reared in lab; 3 larvae and 3 pupal exuviae preserved in alcohol 70\%).

Comments: Specimens were collected with sweep net during day time at sampling site 2. Immature stages were collected at the same site with floating techniques. Larvae were reared in the laboratory until adult emergence. 


\section{Bezzia blantoni Spinelli \& Wirth}

Bezzia blantoni Spinelli \& Wirth 1989: 771 (male, female, pupa; distrib.); Spinelli \& Wirth, 1993: 63 (in list of Argentinean species); Borkent \& Wirth, 1997: 125 (in World catalog); Borkent \& Spinelli, 2000: 60 (in catalog south USA species); Borkent \& Spinelli, 2007: 93 (in Neotropical catalog); Borkent, 2009: 157 (online catalog); Ronderos \& Spinelli, 2009 (larva, pupa).

Distribution: Belize to Costa Rica, Puerto Rico, Argentina (Corrientes, Buenos Aires).

Material examined: Argentina, Buenos Aires prov., Isla Martín García, Arenal Chico, 20-VII-2006, Ronderos-Díaz col., 1 male (reared in lab with larval exuvia), 1 female (reared in lab with pupal exuvia), 3 larvae, 2 pupae; same data except 14-IX-2006, 1 male (reared in lab with pupal exuvia), same data except 11-X-2006, 1 male (reared in lab with pupal exuvia), 2 larvae.

Comments: Immature stages were collected with floating techniques at sampling site 2 . Larvae and pupae were reared in the laboratory until adult emergence.

\section{ACKNOWLEDGMENTS}

We acknowledge Dirección de Administración de Áreas Protegidas y Conservación de la Biodiversidad (Reservas y Monumentos Naturales) Ministerio de Asuntos Agrarios de la Provincia de Buenos Aires for the permission to carry out our research in the island, to Inés César for her suggestions as to Nélida Caligaris for technical assistance.

\section{RESUMEN}

Alrededor de 230 especies de ceratopogónidos han sido registradas o descritas en Argentina, 38 de ellas son conocidas para la provincia de Buenos Aires y sólo una ha sido previamente citada para la Isla Martín García. En este trabajo se presentan los resultados obtenidos a partir de muestreos realizados en seis viajes a la isla durante la primavera de 2005, verano de 2006 y otoño de 2009; se seleccionaron diez sitios de muestreo con ambientes acuáticos permanentes y temporales. La mayoría de los muestreos se llevaron a cabo en cuerpos de agua de diverso origen; algunos de éstos se hallaban cubiertos con vegetación flotante compuesta por Lemna gibba, Lemna minuscule, Salvinia biloba, Salvinia minima, Azolla filiculoides, Limnobium laevigatum, Pistia stratiotes, Spirodela intermedia, Wolffiella oblonga y Wolffia columbiana; mientras que los restantes se realizaron en zonas urbanas y suburbanas. Los adultos se capturaron con redes entomológicas a la salida y puesta del sol y con trampas de luz a intervalos de cuatro a cinco horas por la noche, dependiendo de la disponibilidad de electricidad en la isla. Las larvas y pupas fueron recolectadas con diferentes metodologías según las características de los ambientes estudiados; en el agua libre se capturaron con tamices pequeños o pipetas y micropipetas, y en cuerpos de agua con vegetación se utilizó la técnica de flotación para separar los insectos de las hidrófitas flotantes y arraigadas. Se identificaron trece especies de Ceratopogonidae, 3 de Atrichopogon Kieffer, 3 de Forcipomyia Meigen, 2 de Dasyhelea Kieffer, 4 de Culicoides Latreille, y uno de Bezzia Kieffer, todos ellos representan nuevos registros para la isla.

Palabras clave: Ceratopogonidae, nuevos registros, Isla Martín García, Argentina.

\section{REFERENCES}

Aitken, T.H.G., W.W. Wirth, R.W. Williams, J.B. Davies, \& E.S. Tikasingh. 1975. A review of the bloodsucking midges of Trinidad and Tobago, West Indies (Diptera: Ceratopogonidae). J. Entomol. 44: 101-144.

Armendáriz, L.C. \& I.I. César. 2001. The distribution and ecology of litoral Oligochaeta and Aphanoneura (Annelida) of the Natural and Historical Reserve of Isla Martín García, Río de la Plata river, Argentina. Hidrobiología 463: 207-216.

Barbosa, F.A.S. 1947. Culicoides (Diptera: Heleidae) da regiao neotropica. Ann. Soc. Biol. Pernambuco. 7: $3-30$.

Barbosa, F.A.S. 1952. Novos subsidios para o conhecimento dos Culicoides neotropicos (Diptera: Heleidae). $\mathrm{Ph}$. D. thesis, Universidade do Recife, Recife, Brazil.

Beck, E.C. 1957. Two new species of Culicoides from Florida (Diptera: Heleidae) Fla. Entomol. 40: 103-105.

Blanton, F.S., \& W.W. Wirth. 1979. The sand flies (Culicoides) of Florida (Diptera: Ceratopogonidae). Arthropods of Florida and Neighboring Land Areas; Volume 
10. Florida Department of Agriculture and Consumer Services. Gainesville, Florida.

Borkent, A. 2009. World Species of Biting Midges (Diptera: Ceratopogonidae). (Consultado: 30 noviembre 2009, www.inhs.illinois.edu/research/FLYTREE/ CeratopogonidaeCatalog.pdf).

Borkent, A. \& W.L. Grogan Jr. 2009. Catalog of the New World Biting Midges North of Mexico (Diptera: Ceratopogonidae). Zootaxa 2273: 1-48.

Borkent, A. \& G.R. Spinelli. 2000. Catalog of the New World biting midges south of the United States of America (Diptera: Ceratopogonidae). Contrib. Entomol. Internat. 4: 1-107.

Borkent, A. \& G.R. Spinelli. 2007. Neotropical Ceratopogonidae (Diptera: Insecta), p. 197-198. In J. Adis, J.R. Arias, G. Rueda-Delgado \& K.M. Wantzen (eds.). Aquatic Biodiversity in Latin America (ABLA). Pensoft, Sofia, Moscow.

Borkent, A. \& W.W. Wirth. 1997. World species of biting midges (Diptera: Ceratopogonidae). Bull. Am. Mus. Nat. Hist. 233: 257-258.

Brèthes, J. 1912. Descripción de un nuevo género y especie nueva de Chironomidae (Dipt). An. Mus. Nac. Buenos Aires. 15: 451-453.

Cavalieri, F. \& I. Chiossone. 1966. Sobre el conocimiento actual del género hematófago Culicoides Latreille, 1809, en la Argentina (Diptera: Ceratopogonidae). Physis 26: 145-153.

César, I.I., L.C. Áremendariz \& C. Damborenea. 2001. Ostrácodos (Crustacea) de la Isla Martín García, Río de la Plata, Argentina. Nat. Neotrop. 32: 147-151.

César, I.I. \& R. Liberto. 2008. Ostracoda. ¿Posibles indicadores de deterioro ambiental de la Reserva de Usos Múltiples Isla Martín García? Rev. Ciencia 3: 99-109.

César, I.I., S.M. Martín, B.S. Gullo \& R. Liberto. 2009. Biodiversity and ecology of Hirudinea (Annelida) from the Natural Reserve of Isla Martín García, Río de la Plata, Argentina. Braz. J. Biol. 69: 631-637.

Costa Lima, A. 1937. Chave das especies de Culicoides da região neotropica (Diptera: Ceratopogonidae). Mem. Inst. Oswaldo Cruz 32: 411-422.

Díaz, F., M.M. Ronderos \& G.R. Spinelli. 2005. The immatures of the Neotropical species Culicoides venezuelensis Ortiz \& Mirsa (Diptera: Ceratopogonidae). Trans. Am. Entomol. Soc. 131: 1-20.
Fernández, L.A. \& M.L. López Ruf. 1999. Coleoptera y Heteroptera acuáticos y semiacuáticos de la isla Martín García (Provincia de Buenos Aires). Physis Secc. B. 57: 1-4.

Floch, H \& E. Abonnenc. 1942. Cératopogonides hématophages de la Guyane Francaise. Pub. Inst. Pasteur Guyane Territ. L'Inini 37: 1-10.

Forattini, O.P. 1957. Culicoides da região Neotropical (Diptera, Ceratopogonidae). Arq. Fac. Hig. Saude Pub. Univ. São Paulo 11: 159-526.

Forattini, O.P., E.X. Rabello \& D. Pattoli. 1956. Nota sobre a larva e pupa de Culicoides insignis Lutz, 1913 (Diptera: Ceratopogonidae). Rev. Brasil Ent. 4: 195-198.

Fox, I. 1946. A review of the species of biting midges or Culicoides from the Caribbean Region (Diptera: Ceratopogonidae). Ann. Entomol. Soc. Am. 39: 248-258.

Fox, I. 1948. Hoffmania, a new subgenus in Culicoides (Diptera: Ceratopogonidae). Proc. Biol. Soc. Wash. 61: 21-28.

Fox, I. 1952. Six new Neotropical species of Culicoides (Diptera: Ceratopogonidae). Ann. Entomol. Soc. Am. 45: 364-368.

Fox, I. \& W.A. Hoffman. 1944. New Neotropical biting sand flies of the genus Culicoides (Diptera: Ceratopogonidae). Puerto Rico. J. Pub. Health Trop. Med. 20: $108-111$.

Franca-Rodríguez, M.E. 1963. Primeros estudios sobre los Culicoides (Diptera: Ceratopogonidae. Revta. Urug. Patol. Clin. 1: 65-70.

Ingram, A. \& J.W.S. Macfie. 1931. Ceratopogonidae, p. 155- 232. In Diptera of Patagonia and South Chile. Part II. Fasc. 4, Brit. Mus. (Nat. Hist.), Chile.

Juárez, M.C. 1995. Estatus de residencia y categorización trófica de las aves en la Reserva Natural Isla Martín García, Río de la Plata Superior. Neotropica 41: 83-88.

Lahitte, H.B. \& J.A. Hurrel. 1994. Los Árboles de la Isla Martín García: árboles y arbustos arborescentes (nativos y naturalizados) de la Reserva Natural y Cultural Isla Martín García. CIC, La Plata, Argentina.

Lahitte, H.B. \& J.A. Hurrel. 1996 Plantas hidrófilas de la Isla Martín García (Buenos Aires, República Argentina). CIC, La Plata, Argentina. 
Lahitte, H.B. \& J.A. Hurrel. 1998. Catálogo de las aves de la Isla Martín García (Buenos Aires, Argentina). Serie informe no. 53. CIC, La Plata, Argentina.

Lahitte, H.B., J.A. Hurrel, G. Canda \& C. Trucco Alemán. 1995. Poblaciones de Tupinambis teguixin (SauriaTeidae) de la Isla Martín García (Buenos Aires, Argentina). I Etnobiología y Encuadre MetodológicoCognitivo. Pinaco 2: 45-62.

Lane, J. 1945. Redescricão de Ceratopogonideos Neotropicos (Diptera: Ceratopogonidae). Rev. Entomol. 16: 357-372.

Lane, J. \& I. Ortiz. 1950. Sinonimia en Culicoides (Diptera: Ceratopogonidae). Rev. Sanid. Asist. Soc. 15: 460.

Linley, J.R. 1965. Descriptions of the pupae of Culiciodes barbosai, C. insignis and C. borinqueni (Diptera: Ceratopogonicae). Ann. Entomol. Soc. Am. 58: 57-63.

Lutz, A. 1913. Contribuicao para o estudo das Ceratopogoninas hematofagas do Brazil. Mem. Inst. Oswaldo Cruz. 5: 45-73.

Macfie, J.W.S. 1937. Ceratopogonidae from Trinidad. Ann. Mag. Nat. Hist. Ser. 10, 20: 1-18.

Macfie, J.W.S. 1938. Notes on Ceratopogonidae (Diptera). Proc. R. Entomol. Soc. London (B) 7: 157-166.

Macfie, J.W.S. 1939. A report on a collection of Brazilian Ceratopogonidae (Dipt.). Rev. Entomol. 10: 137-219.

Marino, P.I. \& G.R. Spinelli. 1999. The species groups of the subgenus Forcipomyia (Forcipomyia) in the Neotropics, with a description of a new species of the genualis group (Diptera: Ceratopogonidae). Trans. Am. Entomol. Soc. 125: 445-452.

Marino, P.I. \& G.R. Spinelli. 2002. A revision of the Forcipomyia squamitibia group in the Neotropics with the description of three new species (Diptera: Ceratopogonidae). Ins. Sci. Appl. 22: 307-319.

Messersmith, D.H. 1964. The breeding site of Culicoides debilipalpis Lutz (Diptera: Ceratopogonidae) in Virginia. Mosquito News 24: 339.

Ortiz, I. \& M. Mirsa. 1950. Estudios en Culicoides (Diptera: Ceratopogonidae). Sobre una nueva especie del grupo Haematopotus: Culicoides venezuelensis n. sp. Arch. Venez. Patol. Trop. Parasitol. Med. 2: 137-144.

Philippi, R.A. 1865. Aufzahlung der chilenischen Dipteren. Verh. Kaiser.-König. Zool.-Botan. Gesell. Wien. 15: 595-782.
Ronderos, M.M. \& F. Díaz. 2002. Rearing Culicoides bambusicola (Lutz, 1913) (Diptera, Ceratopogonidae) in lab. Observations and new records. Bol. Mus. Nac. Hist. Nat. Parag. 14: 43-48.

Ronderos, M.M. \& G.R. Spinelli. 1989. The pupa of the bitng midge Culicoides venezuelensis Ortiz \& Mirsa, 1950 (Diptera: Ceratopogonidae). Biota 5: 77-81.

Ronderos, M.M. \& G.R. Spinelli. 1990. Further notes on Culicoides Latreille 1809 from Argentina (Insecta, Diptera, Ceratopogonidae). Iheringia 70: 81-88.

Ronderos, M.M. \& G.R. Spinelli. 1995. Redescripción de Culicoides lahillei y descripción de Culicoides ichesi, n. sp. de la Argentina, Paraguay y Uruguay (Diptera: Ceratopogonidae). Neotrópica 41: 77-81.

Ronderos, M.M. \& G.R. Spinelli. 1998. Las especies de Culicoides en el área de influencia de la represa de Yacyretá (Diptera: Ceratopogonidae). Rev. Soc. Ent. Arg. 57: 79-83.

Ronderos, M.M. \& G.R. Spinelli. 2000. The larva and pupa of Culicoides bambusicola Lutz observed with SEM, and additional notes on the adult (Diptera: Ceratopogonidae).Trans. Am. Entomol. Soc. 126: 133-144.

Ronderos, M.M. \& G.R. Spinelli. 2002. Los Culicoides de la Argentina: clave de especies y nuevos registros (Diptera: Ceratopogonidae), p. 89-95. In D. Salomón (Comp.). Actualizaciones en artropodología sanitaria Argentina. Fundación Mundo Sano, Buenos Aires, Argentina.

Ronderos, M.M. \& G.R. Spinelli 2009. Description of the inmatures of the predaceous midge Bezzia blantoni Spinelli \& Wirthh (Diptera: Ceratopogonidae). Zootaxa 2295: 46-54.

Ronderos, M.M., G.R. Spinelli \& F. Díaz. 2006. Observations on the immatures of Dasyhelea necrophila Spinelli \& Rodríguez in laboratory (Diptera: Ceratopogonidae). Trans. Am. Entomol. Soc. 132: 291-297.

Ronderos, M.M., G.R. Spinelli, H. Huerta \& F. Díaz. 2003a. Immature stages of two Neotropical species of Dasyhelea Kieffer, 1911 (Diptera: Ceratopogonidae) Trans. Am. Entomol. Soc. 129: 295-308.

Ronderos, M.M., G.R. Spinelli, I. Lager \& F. Díaz. 2003b. La importancia sanitaria de los jejenes del género Culicoides (Díptera: Ceratopogonidae) en la Argentina. Rev. Entomol. Vectores 10: 601-612.

Smith, W.W. \& J.H. Varnell Jr. 1967. Hydrogen ion concentration $(\mathrm{pH})$ as related to the occurrence and abundance of tree-holes dwelling Culicoides spp. 
(Diptera: Ceratopogonidae) in northern Florida. Mosquito News 27: 519-521.

Spinelli, G.R. 1982. Estudio sistemático de los Ceratopogonidos de la República Argentina. Tesis No 407, Facultad de Ciencias Naturales y Museo, Universidad Nacional de La Plata, Argentina.

Spinelli, G.R. 1983. Notas sobre Ceratopogonidae de la República Argentina (Diptera: Nematocera). II. Nuevos aportes al conocimiento del género Forcipomyia Meigen. Neotrópica 29: 121-129.

Spinelli, G.R. 1998. Ceratopogonidae, p. 314-326. In J.J Morrone \& S. Coscarón (eds.). Biodiversidad de Artrópodos argentinos. Una perspectiva biotaxonómica. Ediciones Sur, La Plata, Argentina.

Spinelli, G.R. \& E.G. Balseiro. 1982. Observaciones relativas a la actividad nocturna de Ceratopogonidae (Diptera: Nematocera) en la zona de Los Talas (Provincia de Buenos Aires), mediante el empleo de una trampa de luz. Rev. Soc. Ent. Arg. 41: 225-232.

Spinelli, G.R. \& A. Borkent. 2004. New species of Central American Culicoides Latreille (Diptera: Ceratopogonidae) with a synopsis of species from Costa Rica. Proc. Entomol. Soc. Wash. 106: 361-395.

Spinelli, G.R. \& M.E. Martinez. 1991. The genus Culicoides in Uruguay (Diptera: Ceratopogonidae). Insecta Mundi 5: 175-179.

Spinelli, G.R. \& E.A. Rodríguez. 1999. A new species of Dasyhelea from Argentina described as adult, pupae and fourth instar larvae (Diptera: Ceratopogonidae). Neotrópica 45: 59-62.

Spinelli, G.R. \& M.M. Ronderos. 1987. Notas sobre Ceratopogonidae (Diptera: Nematocera) de la Republica Argentina: Nuevos aportes al conocimiento del género Dasyhelea Kieffer. Neotrópica 33: 11-17.

Spinelli, G.R. \& M.M. Ronderos. 1991. Los polvorines del género Culicoides en áreas de influencia de la represa de Salto Grande (Diptera: Ceratopogonidae). Neotrópica 37: 83-94.

Spinelli, G.R. \& M.M. Ronderos. 1997. Description of the male of Culicoides lahillei and revalidation of Culicoides debilipalpis (Diptera: Ceratopogonidae). Trans. Amer. Ent. Soc., Philadelphia 123: 303-309.

Spinelli, G.R. \& M.M. Ronderos. 2005. Ceratopogonidae (Formas hematófagas), p. 61-66. In O. Salomón (ed.). Artrópodos de interés médico en Argentina. Mundo Sano, Buenos Aires, Argentina.
Spinelli, G.R. \& W.W. Wirth. 1986. Clave la identificación de las especies del género Culicoides Latreille presentes al sur de la cuenca Amazónica nuevas citas y notas sinonímicas (Diptera: Ceratopogonidae). Rev. Soc. Ent. Arg. 44: 49-73.

Spinelli, G.R. \& W.W. Wirth. 1989. The Neotropical predaceous midges of the genus Bezzia (Diptera Ceratopogonidae) Part I. The glabra and brevicornis groups. Limnobios 2: 762-778.

Spinelli, G.R. \& W.W. Wirth. 1993. Los Ceratopogonidae de la Argentina (Insecta: Diptera), p. 124. In Z. Castellanos (ed.). Fauna de agua dulce de la República Argentina. Profadu-Conicet Vol. 38, Argentina.

Spinelli, G.R., P.I. Marino \& A. Gaddi. 2010. New records of biting midges from Argentina and Chile (Diptera: Ceratopogonidae). Rev. Soc. Ent. Arg. 69: 127-132.

Spinelli, G.R., P.I. Marino \& M.M. Ronderos. 2005a. The fourth instar larva and pupa $f$ the Neotropical species Forcipomyia (Forcipomyia) rioplatensis Marino \& Spinelli (Diptera: Ceratopogonidae). Proc. Entomol. Soc. Wash. 107: 108-114.

Spinelli, G.R., M.M. Ronderos \& E.G. Balseiro. 1989. Análisis de la actividad diaria de dípteros nematoceros en Punta Lara (Pdo. de Ensenada, Prov. de Buenos Aires). II. Ceratopogonidae, con la descripción de una especie nueva en el genero Atrichopogon. Limnobios 2: 733-737.

Spinelli, G.R., M.M. Ronderos, F. Díaz \& P.I. Marino. 2005b. The bloodsucking biting midges of Argentina (Diptera: Ceratopogonidae). Mem. Inst. Oswaldo Cruz. 100: 137-150.

Trindade, R.L. \& I.S. Gorayeb. 2005. Maruins (Ceratopogonidae: Diptera) do estuário do rio Pará e do litoral do estado do Pará, Brasil. Entomol. Vect. 12: 61-74.

Viana, M.J. 1937. Lista de los insectos de la isla Martín García. Rev. Soc. Ent. Arg. 9: 101-109.

Vitale, G.C., W.W. Wirth \& T.H.G. Aitken. 1981. New species and records of Culicoides reared from arboreal habitats in Panama, with a synopsis of the debilipalpis group (Diptera: Ceratopogonidae). Proc. Entomol. Soc. Wash. 83: 140-159.

Waller, J., R. Lancelot, P.C. Lefevre \& M. Kremer. 1990. Note faunistique sur les Culicoides de Guyane (Diptera: Ceratopogonidae). Rev. Elev. Med. Vet. Pays Trop. 43: 349-364.

Williams, R.W. 1964. Observations on habitats of Culicoides larvae in Trinidad, W.I. (Diptera: Ceratopogonidae). Ann. Ent. Soc. Amer. 57: 462-466. 
Wirth, W.W. 1955. Three new species of Culicoides from Texas (Diptera: Heleidae). J. Washington Acad. Sci. 45: 355-359.

Wirth, W.W. 1960. The correct status of Cotocripus caridei Brèthes, a South American biting midges (Diptera: Ceratopogonidae). Proc. Entomol. Soc. Wash. 62: 44.

Wirth, W.W. 1974. Family Ceratopogonidae, p. 1-89. In N. Papavero (ed.). A catalog of the Diptera of the Americas South of the United Status. Museu de Zoología, Universidade de São Paulo, São Paulo, Brasil.

Wirth, W.W. 1976. Forcipomyia pictoni Macfie and descriptions of two new related species from Florida (Diptera: Ceratopogonidae). Fla. Entomol. 59: 77-84.

Wirth, W.W. \& F.S. Blanton. 1956. Studies in Panama Culicoides VIII. The Neotropical species of the guttatus group of the subgenus Hoffmania (Diptera: Heleidae). Proc. Entomol. Soc. Wash. 58: 305-326.
Wirth, W.W. \& F.S. Blanton. 1959. Biting midges of the genus Culicoides from Panama (Diptera: Heleidae). Proc. U.S. Nat. Mus. 109: 237-482.

Wirth, W.W. \& F.S. Blanton. 1971a. New Neotropical sandflies of the Culicoides debilipalpis group (Diptera: Ceratopogonidae). Proc. Entomol. Soc. Wash. 73: 34-43.

Wirth, W.W. \& F.S.Blanton. 1971b. New species and synonymy of Florida Culicoides (Diptera:Ceratopogonidae). Fla. Entomol. 54: 73-78.

Wirth, W.W. \& F.S. Blanton. 1974. The West Indian sandflies of the genus Culicoides (Diptera: Ceratopogonidae).USDA Tech. Bull. 1474: 1-98.

Wirth, W.W., A.L. Dyce \& G.R. Spinelli. 1988. An atlas of wing photographs, with a summary of the numerical characters of the Neotropical species of Culicoides (Diptera: Ceratopogonidae). Contrib. Entomol. Internat. 25: 1-72. 